\title{
Review
}

Skin

Pharmacology

Physiology
Skin Pharmacol Physiol 2018;31:262-268

DOI: $10.1159 / 000491757$
Received: March 13, 2018

Accepted after revision: July 2, 2018

Published online: August 15, 2018

\section{Epidermal Lamellar Granules}

\author{
Philip Wertz \\ lowa City, IA, USA
}

\section{Keywords}

Lamellar granules - Golgi apparatus · Acylglucosylceramide . ABCA12 - Epidermis · Skin barrier

\begin{abstract}
In the mid-1950s and 1960s, transmission electron microscopes became widely available, leading to many studies of the ultrastructure of various tissues including the epidermis. Most of these studies involved tissue fixation with formaldehyde and postfixation with osmium tetroxide. A few studies employed freeze-fracture electron microscopy. One set of these studies identified a small organelle variously called lamellar granules (LGs), lamellar bodies, membrane-coating granules, cementsomes, and Odland bodies. LGs are round to ovoid in shape, with a diameter of about $200 \mathrm{~nm}$. They have a bounding membrane surrounding a stack of internal lipid lamellae. These small organelles are first seen in the spinous layer and accumulate with differentiation in the granular layer. In the uppermost granular cells, the bounding membrane of the LG fuses into the cell plasma membrane, and the internal contents are extruded into the intercellular space. The initially extruded contents of the LG then rearrange to form the intercellular lamellae of the stratum corneum. In this context, LGs serve as the precursor to the permeability barrier of the skin. Various studies have pro-
\end{abstract}

\section{KARGER}

(C) 2018 S. Karger AG, Basel

E-Mail karger@karger.com

www.karger.com/spp vided evidence that they are derived from the Golgi apparatus, specifically the trans-Golgi. Isolated LGs contain phosphoglycerides, sphingomyelin, and glucosylceramides. The most unusual lipid component is a linoleate-containing glucosylceramide comprising 30- to 34-carbon $\omega$-hydroxyacids. Isolated granules also contain acid hydrolases including glucocerebrosidase, sphingomyelinase, and phospholipase A. They also contain proteases and antimicrobial peptides. Defective LGs have been associated with a number of skin diseases including ichthyotic conditions and defective barrier function. Recently, studies employing cryotransmission electron microscopy have called into question the validity of observations on LGs with more conventional electron microscopic techniques. These studies suggest a continuity of the membrane structure from the Golgi through the intercellular lamellae of the stratum corneum.

(c) 2018 S. Karger AG, Basel

\section{Electron Microscopy of Lamellar Granules: The Classical View}

A typical lamellar granule (LG) with a diameter of about $200 \mathrm{~nm}$ is shown in Figure 1 (review [1]). The internal contents of the LG appear to be pairs of bilayers separated by a central minor electron dense band and 
bounded by major electron dense bands. The distance from the center of a major electron dense band to the center of the next electron dense band is $9.6 \mathrm{~nm}$ [2]. LGs were first described by Selby [3], who thought they were degenerating mitochondria. Subsequently, they were described in detail by Odland [4] and others [5-12]. They were recognized as unique organelles found in keratinizing epithelia and nowhere else. Collectively, these early studies [5-12] established that LGs are membrane-bound and contain one or several tightly packed stacks of bilayer membranes, thought to be derived from flattened and stacked lipid vesicles [1]. LGs first appear in the spinous layer, accumulate in the granular layer, and extrude their internal contents into the intercellular space at the interface between the stratum granulosum and stratum corneum. With slight variation in morphology, LGs are found in the epidermis of mammalian, avian, and reptilian species. Based on transmission electron microscopy (TEM) images, it has been proposed that LGs bud off from the Golgi apparatus [6].

Production of LGs, revealed by TEM, has been used as an indicator of differentiation in keratinocyte cultures [13-21]. Formation of normal LGs has been considered a hallmark of differentiation in organ culture [13], stratified keratinocyte cultures [16, 17, 19-21], and organotypic skin cultures $[14,15,18]$.

\section{Isolated LGs}

In order to determine lipid composition and associated enzymes, several groups have isolated LG-enriched fractions based on their low buoyant density [22-26] or their fairly unique size [ 24,27$]$. In the fractionation based on size, an epidermal homogenate is passed through a series of nucleopore filters of decreasing pore sizes (8.0, 3.0, $1.0,0.8,0.6$, and $0.4 \mu \mathrm{m})$. Since LGs are about $200 \mathrm{~nm}$ in diameter, they will pass through this series of filters while all larger material will be removed. It should be pointed out that LG fractions prepared on the basis of either buoyant density or size are not pure but are enriched in LGs [27].

Direct lipid analyses $[23,24]$ of isolated LGs from rodent and pig epidermis have demonstrated that these organelles contain phospholipids, cholesterol, and glycolipids as major components, and small proportions of fatty acid, cholesterol esters, and ceramides.

The glycolipids are mainly glucosylceramides, the most unusual of which is a linoleate-rich acylglucosylceramide [28-31], which has been implicated in the as-

Epidermal Lamellar Granules

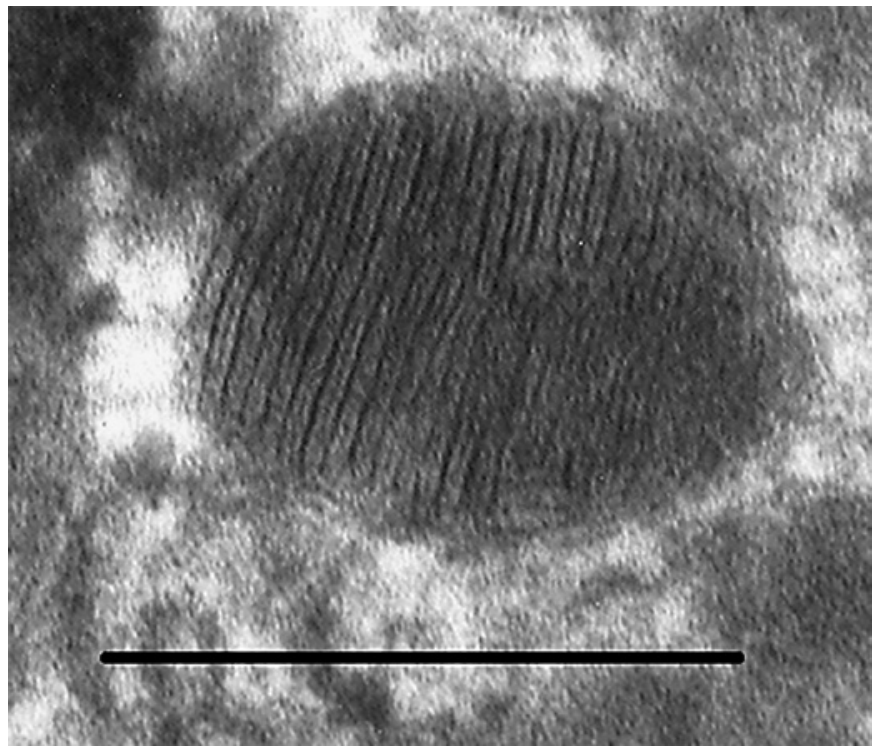

Fig. 1. TEM image of an epidermal LG. Scale bar, $200 \mathrm{~nm}$.

sembly of LGs [32]. This acylglucosylceramide consists of 30 - to 34 -carbon $\omega$-hydroxyacids amide-linked to sphingosine and dihydrosphinmgosine bases, with glucose $\beta$-glycosidically attached to the primary hydroxyl group of the long-chain base and linoleic acid ester-linked to the $\omega$-hydroxyl group (Fig. 2, center).

The 2 strikingly unusual features of this molecule are the very long $\omega$-hydroxyacyl chains, which are long enough to completely span a typical bilayer, and the enrichment in linoleic acid. Linoleic acid is an essential fatty acid without which barrier function deteriorates, and the acylglucosylceramide is the major carrier of linoleic acid in the viable portion of the epithelium. It has been proposed that the $\omega$-hydroxyacyl portion of this molecule does span a bilayer while the linoleate tail inserts into an adjacent region of bilayer, thus riveting the 2 regions of bilayer together at a molecular level [32]. This sort of interaction could promote the flattening and stacking of lipid vesicles in the formation of the LG contents. This "molecular rivet hypothesis" is supported by experiments in which acylglucosylceramide has been shown to promote the aggregation and flattening of synthetic lipid vesicles [33]. It is also supported by the coincidental occurrence of acylglucosylceramide and LGs in all of the keratinizing epithelia and the absence of both acylglucosylceramide and LGs in other tissues, and by the coincidental occurrence of acylglucosylceramide and LGs in the epidermis of terrestrial mammals, birds, and reptiles 
Fig. 2. Epidermal acylglucosylceramide (center) is the precursor of the acylceramide (bottom) and covalently bound $\omega$-hydroxyceramide (top). Amide linkages between the $\omega$-hydroxyacid and the amino group of the long-chain base are on the right, while the ester-linked linoleic acid is on the left.

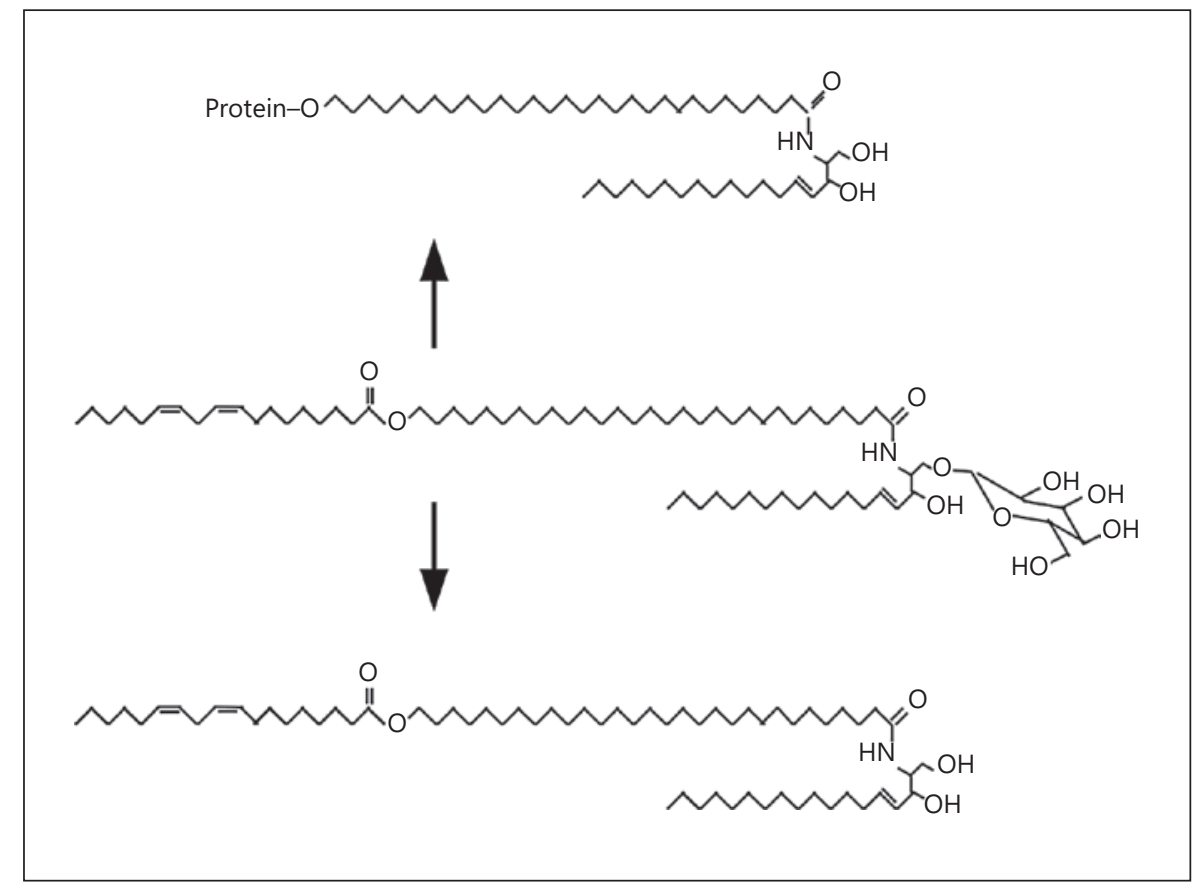

combined with their absence from the epidermis of amphibians and fish [34].

Isolated epidermal LGs have been shown to contain a number of hydrolytic enzymes including $\beta$-glucosidase, acid phosphatase, phospholipase A, sphingomyelinase, carboxypeptidase, and cathepsin B $[24,35]$. They have also been shown to contain ceramide glucosyltransferase, which is considered a biochemical marker for the Golgi [25], and caveolins, which are scaffolding proteins involved in the formation of cholesterol- and sphingolipidrich membrane domains [36]. Sterol sulfatase is not enriched in LGs.

Raymond et al. [26] isolated a human epidermal LGenriched fraction and identified 984 proteins by highthroughput mass spectrometry. Thirty-one of these corresponded to lysosomal components, including lipidprocessing enzymes noted previously. Other identified components include proteolytic enzymes and protease inhibitors, which together could regulate desquamation. One identified protein, CLIP-170, is known to mediate interaction between organelles and microtubules. This may be involved in the movement of the LGs to the apical end of the cell prior to exocytosis of the contents. This study verified the previous findings of Freinkel and Traczyk $[22,35]$ demonstrating the presence of glucocerebrosidase, sphingomyelinase, and prospholipase $\mathrm{A}$ in the LG-enriched fractions. These hydrolytic enzymes are thought to convert glucosylceramides and sphingomyelin into ceramides and to liperate fatty acids from the phosphoglycerides.

Antimicrobial peptides produced in the epidermis are a critical part of innate immunity of the skin $[37,38]$. Defensins and cathelicidins are among the broad-acting antimicrobial peptides produced in the epidermis. They are small (12-50 amino acids), positively charged, and have a hydrophobic domain $[39,40]$. The positive charge allows these molecules to bind to the negatively charged phospholipids. The hydrophobic tail then inserts into the bacterial membrane, causing leakage and cell death. Defensins and cathelicidin were among the peptides identified in the study by Raymond et al. [26]; however, it was possible that these peptides were present as contaminants in the LG preparation. It has been convincingly demonstrated that keratinocytes store cathelicidin in LGs [41]. More research is needed in this area.

\section{Acylglucosylceramide in the Bounding Membrane of LGs and Formation of the Lipid Envelope}

Although there is considerable evidence that acylglucosylceramide plays a role in assembling the internal contents of LGs, it has been estimated that about two-thirds of the LG-associated acylglucosylceramide in the epider- 
mis is in the bounding membrane of the organelle [42]. This is based, in part, on the relative amounts of covalently bound $\omega$-hydroxyceramide and acylceramide found in the stratum corneum. The pool of acylglucosylceramide in the bounding membrane of the LG is introduced to the cell surface when the bounding membrane of the LG fuses into the cell plasma membrane and serves as the precursor of the covalently bound hydroxyceramide (Fig. 2, top). Acylglucosylceramide associated with the internal lamellae of the LG will be extruded and converted into acylceramide (Fig. 2, bottom), which will pass into the intercellular spaces of the stratum corneum.

Given the known sidedness of ceramide glucosyltransferase in the Golgi, it is expected that the glucosyl moiety of acylglucosylceramide in the LG would be on the cytosolic surface, and it is typical for glycolipids in closed vesicular membranes to be oriented with the glucose on the outside. However, this orientation of acylglucosylceramide would position it with the wrong end of the molecule facing the nascent cornified envelope for subsequent attachment of the $\omega$-hydroxyceramide to the envelope proteins, which occurs exclusively via the $\omega$-hydroxyl groups [43]. The action of a flipase at about the time of LG fusion and extrusion could lead to $\omega$-hydroxyceramide with the appropriate orientation. It is speculated that acylglucosylceramide does arrive in the plasma membrane with the glucosyl moiety on the cytoplasmic side, and that the ester-linked fatty acid can be removed through the action of an acid lipase following 2 specific lipoxygenase attacks [44] at the acidic interface between the granular and cornified layers. Flipase action at this time could both expose the glucosyl moiety to $\beta$-glucocerebrosidase at the interface and bring the hydroxyl group into apposition with the nascent cornified envelope proteins to which it becomes attached.

Were acylglucosylceramide molecules in the Golgi to be translocated to orient the glucosyl moiety to the luminal side, additional glycosylation would be expected because the enzymatic mechanism for this is at this location. However, a mechanism that avoids additional glycosylation in the epidermis cannot be ruled out. Flipase action at the time of LG formation would place the glucose moieties on the inside of the LG bounding membrane. Although unusual, this orientation could possibly explain several observations. For one thing, this orientation would place the appropriate $\omega$-hydroxyl end of acylglucosylceramide adjacent to the cornified envelope for lipid envelope formation. The glucose moieties on the inside of the LG could hydrogen bond with water, effectively reducing the activity of free water inside the LG.
This could explain the observation that the LGs are uniquely stable to freezing and thawing [25]. It could also be one factor in explaining the fact that the various hydrolases do not extensively act on their lipid substrates until they are extruded into the extracellular space. Another possibility raised by this orientation of acylglucosylceramide in the LG bounding membrane is that the linoleate tails of the acylglucosylceramide molecules would be in the outer portion of the bounding membrane. As the bounding membrane of the LG comes into close apposition with the inner surface of the cell plasma membrane, the linoleate could extend and insert into the inner leaflet of the plasma membrane. This could locally destabilize the membrane system and facilitate fusion. A similar mechanism has been proposed for the fusion of more conventional phospholipids membranes [45]. Flipases are known in other systems [46, 47] but have not been reported in the epidermis. The above discussion of a possible role for a flipase is speculative; however, one of the most studied flipases is an ATP-binding cassette (ABC) transporter (ABCA4) [47], reminiscent of the LGassociated ABCA12 [48].

\section{LGs and the Golgi Apparatus}

There is considerable evidence that LGs arise from the Golgi apparatus. As noted above, this suggestion was first based on TEM images [7]. A number of biochemical markers of the Golgi have been shown to be associated with LGs including ceramide glucosyltransferase [25], ABCA12 [48, 49], and Rab11 [50].

The Golgi $\mathrm{pH}$ regulator (GPHR) is an anion channel that serves to acidify the lumen of the trans-Golgi and is essential for Golgi function [48, 49, 51]. In the GPHR knockout mouse, LG formation is markedly impaired and barrier function is diminished [52]. In addition to structurally aberrant LGs and increased TEWL, the epidermis of these mice contained less cathelicidin-related antimicrobial peptide (CRAMP).

In one study, the uppermost granular cells were specifically examined [53]. After staining with Nile red or NDB-ceramide, confocal microscopy revealed a tubulereticular membrane system in the apical cytosol of neonatal and adult mouse epidermis. This same trans-Golgi network-like structure was found in the uppermost granular cells in organotypic keratinocyte cultures. When the cultures were challenged by changing the calcium concentration of the medium, it appeared that LGs budded off from the trans-Golgi network-like structure. 


\section{LGs and Disease}

A number of LG defects have been shown to result in skin diseases characterized by diminished barrier function, generally measured as increased TEWL. These diseases are generally characterized by some degree of ichthyosis, the severity of which ranges from mild to lifethreatening.

ABCA12 is an ABC transporter that uses ATP for energy necessary to transport lipids across membranes or into vesicles and is associated with epidermal LGs [49]. Mutations of the gene coding for this transporter are responsible for harlequin ichthyosis [53]. In this condition, the LGs are absent or grossly abnormal [54]. This results in severe reduction of lipids in the intercellular spaces of the stratum corneum and diminished barrier function $[49,55]$. Cornified envelope proteins and keratins are not affected [56]. Other mutations of this same gene may result in lamellar ichthyosis or congenital ichthyosiform erythroderma $[55,57]$.

Mutations of the gene VPS33B results in arthrogryptosos, renal disfunction and cholestasis syndrome (ARC). Patients with ARC suffer severe ichthyosis. In this condition, LGs have abnormal morphology and exocytosis is defective $[58,59]$.

Hyperkeratosis in atopic dermatitis lesions has been attributed to incomplete secretion of the serine protease KLK 7 from LGs and upregulation of the serine protease inhibitor LEKTI [60].

A mutation of CGI-58 protein has been shown to cause Dorfman-Chanarin syndrome, one characteristic of which is ichthyosis. The CGI-58 protein is involved in triglyceride breakdown and, with this, the mutant protein lipid droplets accumulate in the cytosol of the keratinocyte. Electron microscopy has revealed that the LG contents are abnormal, and it has been suggested that this defect may lead to the ichthyotic condition [61]. Likewise, a specific lipoxygenase mutation has been shown to cause incomplete LG secretion, leading to congenital ichthyosaform erythroderma [62]. Both Dorfman-Chanarin syndrome and congenital ichthyosaform erythroderma are autosomal recessive diseases.

Anything that significantly alters LG contents or interferes with their exocytosis is likely to result in diminished permeability barrier function, attributed to decreased or inappropriate lipids in the intercellular spaces of the stratum corneum, defective desquamation due to failed or diminished delivery of proteases required for desquamation, or increased susceptibility to infection due to diminished delivery of antimicrobial peptides. In a number of skin diseases with impaired barrier function, the barrier has been at least partially restored by topical application of ceramides or combinations of ceramides plus cholesterol and fatty acids [63-71].

\section{An Alternative View}

In 2003, Norlen et al. [72] published a cryo-TEM study of skin barrier formation in which they described a tubuloreticular network, similar to that described by Elias et al. [53], in the secretory granular cell after using a microwave-enhanced fixation technique. There were no discrete LGs. This was further elaborated upon by den Hollander et al. [73].

Ishida-Yamamoto et al. [74] imaged a number of LG markers by confocal laser scanning microscopy and by immunoelectron microscopy. These markers included glucosylceramides, cathepsin $\mathrm{D}$, corneodesmosin, kallikrein, and KLK8. They concluded that their results were consistent with a tubuloreticular system rather than discrete LGs.

The findings of Elias et al. [53] suggest that, in an unstimulated state, the tubuloreticular network predominates and individual LGs may be sparse, at best. However, under stress to stimulate lipid secretion, individual LGs may bud off from this network. Some kinds of chemical fixation may acutely provide such a stress.

\section{Conclusion}

Traditional electron microscopic studies, including TEM and the freeze-fracture method, have revealed LGs as small organelles in keratinizing epithelia. They are round to ovoid in shape with a diameter of about $200 \mathrm{~nm}$ and consist of a unit-bounding membrane surrounding stacks of internal membranous disks. They first appear in the spinous layer, increase throughout the granular layer, and extrude their contents at the boundary between the granular layer and the stratum corneum. Studies of isolated LGs support their origin from the Golgi. Isolated LGs contain sphingomyelin and glucosylceramides, and also the hydrolytic enzymes necessary to convert these lipids into ceramides. LGs also deliver proteolytic enzymes necessary for desquamation and antimicrobial peptides to the intercellular space. Mutations of ABCA12 result in abnormal LGs and lead to either harlequin ichthyosis, lamellar ichthyosis, or congenital ichthyosiform erythroderma. Several other mutations that alter LGs also 
result in ichthyotic conditions. Recent work with cryoTEM suggests that LGs may not exist as discreet organelles. Instead, it is proposed that there is membrane continuity from the Golgi through the intercellular spaces of the stratum corneum.

Additional work is needed to determine whether the view of LGs based on traditional TEM and freeze-fracture electron microscopy is valid or needs to be replaced with the continuous membrane model based on cryo-TEM.

\section{Acknowledgements}

The author would like to acknowledge Nancy Wertz for editorial assistance in the preparation of the manuscript.

\section{Disclosure Statement}

The author has no conflict of interest with respect to this work.

\section{References}

1 Landmann L: The epidermal permeability barrier. Anat Embryol 1988;178:1-13.

2 Madison KC, Swartzendruber DC, Wertz PW, Downing DC: Presence of intact intercellular lamellae in the upper layers of the stratum corneum. J Invest Dermatol 1987;88: 714-718.

3 Selby CC: An electron microscope study of thin sections of human skin. II. Superficial layers of footpad epidermis. J Invest Dermatol 1957;29:131-149.

4 Odland GF: A submicroscopic granular component in human epidermis. J Invest Dermatol 1960;34:11-15.

5 Frei JV, Sheldon H: A small granular component of the cytoplasm of keratinizing epithelia. J Biophys Biochem Cytol 1961;11:719724.

6 Farbman AI: Electron microscope study of a small cytoplasmic structure in rat oral epithelium. J Cell Biol 1964;21:491-495.

7 Matoltsy AG, Parakal PF: Membrane coating granules of keratinizing epithelia. J Cell Biol 1965;24:297-307.

8 Hashimoto K: Ultrastructure of the human toenail. Cell migration, keratinization and formation of the intercellular cement. Arch Dermatol Forsch 1971;240:1-22.

9 Elias PM, NcNutt NS, Friend DS: Membrane alterations during cornification of mammalian epithelia. A freeze-fracture, tracer and thin-section study. Anat Rec 1977;189:577594.

10 Wolff-Schreiner E: Ultrastructural cytochemistry of the epidermis. Int J Dermatol 1977;16: 77-102.

11 Hayward AF: Membrane coating granules. Int Rev Cytol 1979;59:97-127.

12 Landmann L: LGs in mammalian, avian and reptilian epidermis. IJ Ultrastruc Res 1980;72: 245-263.

13 Bickenbach JR, Holbrook KA: Proliferation of human embryonic and fetal epidermal cells in organ culture. Am J Anat 1986;177:97-106.

14 Madison KC, Swartzendruber DC, Wertz PW, Downing DT: Lamellar extrusuion and stratum corneum intercellular lamellae in murine keratinocyte cultures. J Invest Dermatol 1988;90:110-106.
15 Madison KC, Swartzendruber DC, Wertz PW, Downing DT: Murine keratinocyte cultures grown at the air/liquid interface synthesize stratum corneum lipids and "recycle" linoleate during differentiation. J Invest Dermatol 1989;93:10-17.

16 Bernstein LI, Vaughan FL, Bernstein IA: Stratified cornified primary cultures of human keratinocytes grown on microporous membranes at the air-liquid interface. J Derm Sci 1990;1:173-181.

17 Rosdy M, Clauss LC: Terminal epidermal differentiation of human keratinocytes grown in chemically defined medium on inert filter substrates at the air-liquid interface. J Invest Dermatol 1990;95:409-414.

18 Sando GN, Howard EJ, Madison KC: Induction of ceramide glucosyltransferase activity in cultured human keratinocytes. Correlation with culture differentiation. J Biol Chem 1996;271:22044-22051.

19 Poumay Y, Dupont F, Marcoux S, LeclercqSmekens M, Herin M, Coquette A: A simple reconstructed human epidermis: preparation of the culture model and utilization in in vitro studies. Arch Dermatol Res 2004;296:203-211.

20 Yamanaka Y, Akiyama M, Sugiyama-Nakagiri Y, Sakai K, Goto M, McMillan JR, Ota M, et al: Expression of the keratinocyte lipid transporter $\mathrm{ABCA} 12$ in developing and reconstituted human epidermis. Am J Pathol 2007;16: 43-52.

21 Qiu J, Zhong M, Chen D, Huang X, Chen J, Chen M, et al: Establishment and characterization of a reconstituted Chinese human epidermis model. Int J Cosmet Sci 2016;38:60-67.

22 Freinkel RK, Traczyk TN: A method for partial purification of LGs from fetal rat epidermis. J Invest Dermatol 1981;77:478-482.

23 Wertz PW, Downing DT, Freinkel RK, Traczyk TN: Sphingolipids of the stratum corneum and LGs of fetal rat epidermis. J Invest Dermatol 1984;83:193-195.

24 Grayson S, Johnson-Winegar AG, Wintraub BU, Isseroff RR, Epstein EH, Elias PM: Lamellar body-enriched fractions from neonatal mice: preparative techniques and partial characterization. J Invest Dermatol 1985;85:289_ 294.
25 Madison KC, Sando GN, Howard EJ, True CA, Gilbert D, Swartzendruber DC, Wertz PW: LG biogenesis: a role for ceramide glucosyltransferase, lysosomal enzyme transport, and the Golgi. J Invest Dermatol Symp Proc 1998;3:80-86.

26 Raymond AA, Gonzales de Peredo A, Stella A, Ishida-Yamamoto A, Bouyssie D, Serre G, et al: Lamellar bodies of human epidermis. Mol Cell Proteomics 2008;7:2151-2175.

27 Grayson S, Johnson-Winegar AD, Elias PM: Isolation of lamellar bodies from neonatal mouse epidermis by selective sequential filtration. Science 1983;221:962-964.

28 Gray GM, White RJ, Majer JR: 1-(3-O-acyl)- $\beta$ glucosyl-N-dihydroxypentatriacontadienoylsphingosine, a major component of the glucosylceramides of pig and human epidermis. Biochim Biophys Acta 1978;528:127-137.

29 Wertz PW, Downing DT: Acylglucosylceramides of pig epidermis: structure determination. J Lipid Res 1983;24:753-758.

30 Abraham W, Wertz PW, Downing DT: Linoleate-rich acylglucosylceramides from pig epidermis: structure determination by proton magnetic resonance. J Lipid Res 1985;26:761766 .

31 Bowser PA, Nugteren DH, White RJ, Houtsmuller UMT, Prottey C: Identification, isolation and characterization of epidermal lipids containing linoleic acid. Biochim Biophys Acta 1985;834:419-428.

32 Wertz PW, Downing DT: Glycolipids in mammalian epidermis: structure and function in the water barrier. Science 1982;217: 1261-1262.

33 Landmann L, Wertz PW, Downing DT: Acylglucosylceramide causes flattening and stacking of liposomes: an analogy for assembly of the epidermal permeability barrier. Biochim Biophys Acta 1984;778:412-418.

34 Wertz PW: Lipids of keratinized tissues; in Matoltsy GA, Richards KS, Bereiter-Hahn J (eds): Biology of the Integument, vol 2. Berlin, Springer, 1986, pp 815-823.

35 Freinkel RK, Traczyk TN: Acid hydrolases of the epidermis: subcellular localization and relationship to cornification. J Invest Dermatol 1983;80:441-446. 
36 Sando GN, Zhu H, Weis JM, Richman JT, Wertz PW, Madison KC: Caveolin expression and localization in human keratinocytes suggests a role in LG biogenesis. J Invest Dermatol 2003; 120:531-541.

37 Weinberg A, Krisanaprakornkit S, Dale BA: Epithelial antimicrobial peptides: review and significance for oral applications. Crit Rev Oral Biol Med 1998;9:399-414.

38 Afshar M, Gallo RL: Innate immune defense system of the skin. Vet Dermatol 2013;24:3238.

39 Takahashi T, Gallo RL: The critical and multifunctional roles of antimicrobial peptides in dermatology. Cermatol Clin 2017;35:39-50.

40 Brandwein M, Bentwich Z, Steinberg D: Endogenous antimicrobial peptide expression in response to bacterial epidermal colonization. Front Immunol 2017;8:1637.

41 Braff MH, Di Nardo A, Gallo RL: Keratinocytes store the antimicrobial peptide cathelicidin in lamellar bodies. J Invest Dermatol 2005;124:394-400.

42 Wertz PW: Lipids and barrier function of the skin. Acta Derm Veneriol Supp 2000:208:711.

43 Stewart ME, Downing DT: The omega-hydroxyceramides of pig epidermis are attached to corneocytes solely through omega-hydroxyl groups. J Lipid Res 2001;42:1105-1110.

44 Zheng Y, Yin H, Boeglin WE, Elias PM, Crumrine D, Beier DR, Brash AR: Lipoxygenases mediate the effect of essential fatty acid in skin barrier formation. J Biol Chem 2011;286: 24046-244056.

45 Holopainen JM, Lehtonen JY, Kinnunen PK: Evidence for the extended phospholipids conformation in membrane fusion and hemifusion. Biophys J 1999;76:2111-2120.

46 Banerjee T, Kuypers FA: Reactive oxygen species and phosphatidylserine externalization in murine sickle red cells. Br J Haematol 2004 124:391-402.

47 Sullivan JM: Focus on molecules: ABCA4 $(\mathrm{ABCAR})$ - an import-directed photoreceptor retinoid flipase. Exp Eye Res 2012;89:602603.

48 Sakai K, Aklyama M, Suglyama-Nakagiri Y, Mamillan JR, Sawamura D, Shimizu H: Localization of ABCA12 from Golgi apparatus to LGs in human upper epidermal keratinocytes. Exp Dermatol 2007;16:920-926.

49 Weicz OA: Acidification and protein traffic. Int Rev Cytol 2003;226:259-319.

50 Ishida-Yamamoto A, Kishibe M, Takahash $\mathrm{H}$, Izuka $\mathrm{H}$ : Rab11 is associated with epidermal LGs. J Invest Dermatol 2007;127:21662170.

51 Akiyama M: The roles of ABCA12 in keratinocyte differentiation and lipid barrier formation in the epidermis. Dermatoendocrinology 2011;3:107-112.
52 Tarutani M, Nakalima K, Uchida Y, Takaishi M, Goto-Inoue N, Ikawa M, et al: GPHR-dependent functions of the Golgi apparatus are essential for the formation of LGs and the skin barrier. J Invest Dermatol 2012;132:20192025.

53 Elias PM, Cullander C, Mauro T, Rassner U, Kamuves L, Brown BE, Menon GK: The secretory granular cell: the outermost granular cell as a specialized secretory cell. J Invest Dermatol Symp Proc 1998;3:87-100.

54 Dale BA, Holbrook KA, Fleckman P, Kimball JR, Brumbaugh S, Sybert VP: Heterogeneity in harlequin ichthyosis, an inborn error of epidermal keratinization: variable morphology and structural protein expression and a defect in LGs. J Invest Dermatol 1990;94:618.

55 Milner ME, O'Guin WM, Holbrook KA, Dale BA: Abnormal LGs in harlequin ichthyosis. J Invest Dermatol 1992;99:824-829.

56 Feingold KR, Denda M: Regulation of permeability barrier homeostasis. Clin Dermatol 2012;30:263-268.

57 Akiyama M, Yoneda K, Kim SY, Koyama H, Shimizu H: Cornified cell envelope proteins and keratins are normally distributed in harlequin ichthyosis. J Cutam Pathol 1996;23: 571-575.

58 Richard G: Autosomal recessive congenital ichthyosis; in Adam MP, Ardinger $\mathrm{HH}$ Pagon RA, Wallace SE, Bean LJH, Stephens K, Amemiya A (eds), GeneReviews. The University of Washington, Seattle, 1993-2018. 2001. https://www.ncbi.nlm.nih.gov/books/ NBK1420/ (updated 18 May 2017).

59 Hershkovitz D, Mandel H, Ishida-Yamamoto A, Chefetz I, Hino B, Luder A, et al: Defective LG secretion in arthrogryptosos, renal disfunction, and cholestasis syndrome caused by a mutation in VPS33B. Arch Dermatol 2008; 144:334-340.

60 Rogerson C, Gissen P: VPS33B and VIPAR are essential for epidermal lamellar body biogenesis and function. Biochim Biophys Acta 2018; 1864:1609-1621.

61 Igawa S, Kishibi M, Minami-Hori M, Honma $\mathrm{M}$, Tsujimura $\mathrm{H}$, Ishikawa J, et al: Incomplete KLK7 secretion and upregulated LEKTI expression underlie hyperkeratotic stratum corneum in atopic dermatitis. J Invest Dermatol 2017;137:614-620.

62 Akiyama M, Sawamura D, Nomura Y, Sugawara M, Shimizu H: Truncation of CGI-58 protein causes malformation of LGs resulting in ichthyosis in Dorfman-Chanarin syndrome. J Invest Dermatol 2003;121:10291034 .

63 Akiyama M, Sakai K, Yanagi T, Tabata N, Yamada M, Shimizu H: Partially disturbed LG secretion in mild congenital ichthyosiform erythroderma with ALOX12B mutations. Br J Dermatol 2010;163:201-204.
64 Zettersten EM, Ghadially R, Feingold KR, Crumrine D, Elias PM: Optimal ratios of topical stratum corneum lipids improve barrier recovery in chronically aged skin. J Am Acad Dermatol 1997;37:403-408.

65 Chamin SL, Kao J, Frieden IJ, Sheu MY, Fowler AJ, Fluhr JW, et al: Ceramide-dominant barrier repair lipids alleviate childhood atopic dermatitis: changes in barrier function provide a sensitive indicator of dosease activity. J Am Acad Dermatol 2002;47:198-208.

66 Vavrova K, Hrabalek A, Mac-Mary S, Humbert P, Muret P: Ceramide analogue 14S24 selectively recovers perturbed human skin barrier. Br J Dermatol 2007;157:704-712.

67 Daehnhardt D, Daehnhardt-Pfeffer S, Schulte-Walter J, Neubourg T, Hanisch E, Schmetz $\mathrm{C}$, et al: The influence of two different foam creams on skin barrier repair of foot xerosis: a prospective, double-blinded, randomized, placebo-controlled intra-individual study. Skin Pharmacol Physiol 2016;29:266-272.

68 Ma L, Li P, Tang J, Guo Y, Shen C, Chang J, Kerrouche N: Prolonging time to flare in pediatric atopic dermatitis: a randomized, investigator-blinded, controlled, multicenter clinical study of ceramide-containing moisturizer. Adv Ther 2017;34:2601-2611.

69 Neubert RH, Sonnenberger S, Dobner B, Gray CW Jr, Barger KN, Sevi-Maxwell K, et al: Controlled penetration of a novel dimeric ceramide into and across the stratum corneum using microemulsions and various types of semisolid formulations. Skin Pharmacol Physiol 2016;130-134.

70 Danby SG, Brown K, Higgs-Bayliss T, Chittock J, Albenali L, Cork MJ: The effect of an emollient containing urea, ceramide NP, and lactate on skin barrier structure and function in older people with dry skin. Skin Pharmacol physiol 2016;29:135-147.

71 Tessema EN, Gebre-Mariam T, Neubert RHH, Wohlrab J: Potential applications of phyto-derived ceramides in improving epidermal barrier function. Skin Pharmacol Physiol 2017;30:115-138.

72 Norlen l, Al-Amoudi A, Dubochet J: A cryotransmission electron microscopy study of skin barrier formation. J Invest Dermatol 2003; 120:555-560.

73 Den Hollander L, Han H, de WinterM, Daneholt B, Norlen L: Skin lamellar bodies are not discrete vesicles but part of a tubuloreticular network. Acta Derm Venereol 2016;96:303308.

74 Ishida-Yamamoto A, Simon M, Kishibe M, Miyauchi Y, Takahashi H, Yoshida S, et al: Epidermal LGs transport different cargoes as distinct aggregates. J Invest Dermatol 2004; 122:1134-1144. 